\title{
Imaging biomarkers in oncology: we can get more from what we see
}

\author{
Arturo Chiti
}

Received: 14 December 2014 / Accepted: 17 December 2014 /Published online: 23 January 2015

(C) Springer-Verlag Berlin Heidelberg 2015

Medical imaging has evolved from a way to see things beneath the skin of a living body to a sophisticated way to visualize the behaviour of biological processes under changing conditions and in different environments. Moreover, imaging now aims not only to describe processes, but also to provide a key to understanding what is behind a particular process in a particular organism. The integration of biomarkers into clinical trials is of paramount importance, particularly in a time when changes are required to establish new, faster and lighter ways to validate drugs, surgical procedures, radiation therapy schemes and medical devices.

Another important shift in the way we evaluate drugs and procedures is the choice of endpoints. More importance is given to those endpoints that reflect the real benefit to a patient: overall survival and disease-free survival. Along with these endpoints, an objective assessment of the quality of life is increasingly important. The question is simple: under what conditions can one accept some more weeks of life? Is it better to survive some more weeks or to die earlier with dignity? Is the high cost of medical care in the last months of an individual's life worth the cost paid by the community? Of course these are ethical considerations that vary among different cultures and different individuals and are beyond the scope of this brief introduction.

Most research is focusing on validating biomarkers extracted from tissue or blood samples. These samples can be stored and a single sample can be evaluated for the presence of several biomarkers now and in the future, including biomarkers that might be discovered in the next years. Moreover, samples from many individuals can be collected and analysed together,

A. Chiti $(\bowtie)$

Nuclear Medicine, Humanitas Research Hospital, Rozzano, MI, Italy

e-mail: arturo.chiti@humanitas.it allowing direct calculation of data from an entire population and cost reduction. The limitation of these biomarkers is related to the fact that they are extracted from a single or very few sites of disease. This means that the results of their evaluation are related to only a small fraction of the whole disease affecting a particular individual.

Imaging biomarkers has the disadvantage of allowing evaluation of just one or two processes at a time (e.g. size and glucose consumption of a primary lung cancer) in just a single patient. Moreover, they are often expensive, because they require the implementation of sophisticated imaging technology. On the other hand, imaging biomarkers has several advantages: the procedures involved are noninvasive; they are able to show several disease sites at the same time, allowing evaluation of the whole disease burden; and they allow evaluation of the heterogeneity of biomarker expression at a particular site (e.g. tissue using glucose and necrotic tissue in a primary lung cancer).

Many technologies are being used as imaging biomarkers: plain radiography, CT, MRI, SPECT, PET and optical imaging. In particular, when using MRI and PET there are several different sequences or radiopharmaceuticals that represent different biomarkers, extracted with the same technology.

Quantification of biomarkers is of paramount importance when a biomarker has to be used in patient management. Much effort is being applied to the validation of quantitative biomarkers in oncology, again with a special focus on MRI and PET. Quantitative assessment must be reproducible, and this implies that, before a biomarker can be validated, the technology used to extract it must be standardized. Many initiatives are running with the goal of standardizing imaging modalities in clinical trials. Among these programmes, we are proud of what EANM has achieved through the EARL accreditation programme for FDG PET/CT. Other 
standardization programmes developed by EANM will be available in the next years, covering the introduction of new radiopharmaceuticals and new technologies in clinical trials.

In this year's themed issue of the European Journal of Nuclear Medicine and Molecular Imaging, several experts describe how imaging biomarkers can be derived and used in clinical trials. Two papers focusing, respectively, on PET radiopharmaceuticals and MRI and CT used as imaging biomarkers open the issue. They are followed by an overview of the use of imaging biomarkers in the preclinical setting and by papers dedicated to five of the main diseases, or groups of diseases, in which imaging biomarkers are used. We have tried to design a comprehensive themed issue that introduces a different way to view imaging, going beyond the concept of pure diagnostic imaging, and we hope that readers will find it useful.

This is a fast-evolving field and we are aware that knowledge and technologies evolve rapidly. We therefore expect that this themed issue will provide a clearer idea of the use imaging biomarkers in oncology and will help build the basis for the understanding of the evolution of this field of imaging. 